\title{
A MODEL OF FUZZY TOPOLOGICAL RELATIONS FOR SIMPLE SPATIAL OBJECTS IN GIS
}

\section{Um modelo de relações topológicas fuzzy para objetos espaciais em GIS}

\author{
BO LIU $1,2,3$ \\ DAJUN LI ${ }^{2}$ \\ JIAN RUAN ${ }^{2}$ \\ LIBO ZHANG 4 \\ LAN YOU $5,1^{*}$ \\ HUAYI WU 1
}
${ }^{1}$ State Key Laboratory of Information Engineering in Surveying, Mapping and Remote Sensing, Wuhan University, 129 Luoyu Road, Wuhan 430079, China
${ }^{2}$ Faculty of Geomatics, East China Institute of Technology, 418\#Guanglan Road, Nanchang 330013, PR, China
${ }^{3}$ Key laboratory of watershed ecology and geographical environment monitoring, National
Administration of Surveying, Mapping and Geoinformation, 418\#Guanglan Road, Nanchang 330013, PR, China

${ }^{4}$ National Quality Inspection and Testing Center for Surveying and Mapping Products, 28Lianhuachi West Road, Hai dian District, Beijing 100830, PR, China

${ }^{5}$ Faculty of Computer Science and Information Engineering, Hubei University,

368Youyi Avenue, Wuhan 430062, PR, China

bliu@whu.edu.cn; djli@ecit.cn; jruan@ecit.cn; 37856062@qq.com; youlan1025@gmail.com;wuhuayi@whu.edu.cn

\begin{abstract}
:
The goal of this paper is to present a new model of fuzzy topological relations for simple spatial objects in Geographic Information Sciences (GIS). The concept of computational fuzzy topological space is applied to simple fuzzy objects to efficiently and more accurately solve fuzzy topological relations, extending and improving upon previous research in this area. Firstly, we propose a new definition for simple fuzzy line segments and simple fuzzy regions based on computational fuzzy topology. And then, we also propose a new model to compute fuzzy topological relations between simple spatial objects, an analysis of the new model exposes:(1) the topological relations of two simple crisp objects; (2) the topological relations between one simple crisp object and one simple fuzzy object; (3) the topological relations between two simple fuzzy objects. In the end, we have discussed some examples to demonstrate the validity of the new model, through an experiment and comparisons of existing models, we showed that the proposed method can make finer distinctions, as it is more expressive than the existing fuzzy


models.

Keyword: Topological Relation; Simple Spatial Objects; Fuzzy Topology; Model; GIS.

\section{Resumo:}

O objetivo deste artigo é apresentar um novo modelo de relações topológicas fuzzy para objetos espaciais simples em Sistemas de Informações Geográficas (SIG). Aplica-se o conceito de espaço topológico fuzzy a objetos fuzzy simples para resolver as relações topológicas de modo mais eficiente e acurado. Inicialmente é proposta uma nova definição para segmentos de linha fuzzy e para regiões fuzzy com base na topologia computacional. Em seguida propõe-se um novo modelo para calcular as relações topológicas fuzzy entre os objetos espaciais. A análise do novo modelo aponta: (1) relações topológicas de dois objetos "crisp"; (2) relações topológicas entre um objeto "crisp" e um objeto fuzzy; (3) relações topológicas entre dois objetos "crisp". Finalmente, discutem-se alguns exemplos para demonstrar a validade do modelo, por meio de um experimento e comparações entre modelos existentes. É possível demonstrar que o método proposto pode realizar distinções mais precisas e á mais expressivo do que os modelos fuzzy existentes.

Palavras-chave: Relações Topológicas; Objetos Espaciais Simples; Topologia Fuzzy; Modelo; GIS.

\section{Introduction}

Geographical information sciences (GIS) commonly deal with geographical phenomena modeled by crisp points, lines, and regions, features which are clearly defined or have crisp boundaries. However, geospatial data are always uncertain or fuzzy due to inaccurate data acquisition, incomplete representation, dynamic change, and the inherent fuzziness of geographical phenomena itself. In GIS, many studies have been devoted to modeling topological relations, specifically the modeling of fuzzy topological relations between simple spatial objects. Topology is a fundamental challenge when modeling the spatial relations in geospatial data that includes a mix of crisp, fuzzy and complex objects. Two mechanisms, the formalization and reasoning of topological relations, have become popular in recent years to gain knowledge about the relations between these objects at the conceptual and geometrical levels. These mechanisms have been widely used in spatial data query (Egenhofer, 1997, Clementini et al., 1994), spatial data mining (Clementini et al., 2000), evaluation of equivalence and similarity in a spatial scene (Paiva, 1998), and for consistency assessment of the topological relations of multi-resolution spatial databases (Egenhofer et al., 1994, 1995; Du et al., 2008a, b). Diloet al. (2007) defined several types and operators for modeling spatial data systems to handle fuzzy information. Shi et al. (2010) and Liu et al. (2011) developed a new object extraction and classification method based on fuzzy topology.

However, the fuzzy topological relationships themselves must be modeled due to the existence of indeterminate and fuzzy boundaries between spatial objects in GIS. Fuzzy topology theory can potentially be applied to the modeling of fuzzy topological relations among spatial objects.

To date, many models have been designed to formalize fuzzy topological relations between simple spatial objects these models provide a framework to conceptually describe the topological Bol. Ciênc. Geod., sec. Artigos, Curitiba, v. 21, no 2, p.389-408, abr-jun, 2015. 
relations between two regions and can be considered as an extension of the crisp case. They can be implemented on spatial databases at less cost than other uncertainty models and are useful when managing, storing, querying, and analyzing uncertain data. For example, Egenhofer and Franzosa (1991a, 1994, 1995) and Winter (2000) modeled the topological relations between two spatial regions in two dimensional space (2-D) based on the 4-intersection model and ordinary point set theory. Li et al. (1999), Long and Li (2013) produced a Voronoi-based 9-intersection model based on Voronoi diagrams. Cohn et al. $(1996,1997)$ discovered forty-six topological relations between two regions with indeterminate boundaries based on Region Connection Calculus (RCC) theory (Randell et al., 1992). Clementini and Di Felice (1996a, b, 1997) used extended 9-intersection model to classify forty-four topological relations between simple regions with broad boundaries. The extended 9-intersection model substantially agrees with the RCC model, though the former removes two relations considered as invalid in the geographical environment. The extended 9-intersection model can be extended to represent topological relations between objects with different dimensions, like regions and lines, while the RCC model can only be applied to relations between regions. Tang and Kainz (2002), Tang et al. (2005), Tang (2004) applied fuzzy theory and a 9-intersection matrix and discovered forty-four topological relations between two simple fuzzy regions. Shi and Liu (2004) discussed fuzzy topological relations between fuzzy spatial objects based on the theory of fuzzy topology. Du et al. (2005a, b) proposed computational methods for fuzzy topological relations description, as well as a new fuzzy 9-intersection model. Liu and Shi (2006, 2009), Shi and Liu(2007) defined a computational fuzzy topology to compute the interior, boundary, and exterior parts of spatial objects, and based on the definition, Liu and Shi(2009) proposed a computational 9-intersection model to compute the topological relations between simple fuzzy region, line segment and fuzzy points, but the model did not give the topological relations between two simple fuzzy regions, and did not compute the topological relations between one simple fuzzy spatial object and one simple crisp spatial object.

To further investigate the application of fuzzy topological relations in GIS, on the basis of previous researches, this study develops a new model of describing the fuzzy topological relations for simple fuzzy objects. The new model not only computes the topological relations between simple crisp spatial objects, but also computes the topological relations between simple fuzzy spatial objects.

The remainder of this paper is organized as follows. In section 2, some basic concepts of fuzzy topology, computational fuzzy topology and the definitions of simple fuzzy spatial objects in GIS are detailed; In the Section 3, the new definition of simple fuzzy spatial objects is presented, and a new model of fuzzy topological relations for simple fuzzy spatial objects is proposed; In Section 4, some examples are discussed to validate the proposed method. Finally, some conclusions are drawn in Section 5.

\section{A Brief Summary of Computational Fuzzy Topology}

In this section, coherent fuzzy topologies, induced by interior and closure operators (Liu and Shi, 2006, 2009; Shi and Liu, 2007; Liu and Luo, 1997), are reviewed. Mathematically, point set 
topology is the fundamental theory for modeling topological relations between simple crisp spatial objects in GIS. By extension, fuzzy topology is a generalization of ordinary topology that introduces the concept of membership value and can be adopted for modeling topological relations between spatial objects with uncertainties. Zadeh (1965) introduced the concept of fuzzy sets, and fuzzy set theory. Fuzzy topology was further developed based on the fuzzy sets (Chang, 1968; Wong, 1974; Wu and Zheng, 1991; Liu and Luo, 1997).Liu and Shi (2006, 2009), and Shi and Liu (2007) defined a computational fuzzy topology to compute the interior, boundary and exterior of spatial objects. The computation is based on two operators, the interior operator and the closure operator. Each interior operator corresponds to one fuzzy topology and that each closure operator also corresponds to one fuzzy topology (Liu and Luo, 1997). The research detailed in this paper extends this work by defining fuzzy spatial objects. However, it is important to review basic concepts in fuzzy set theory as well as simple fuzzy objects in GIS.

\subsection{Basic Concepts}

We focus on the two-dimensional Euclidean plane $R^{2}$, with the usual distance and topology. Fuzzy topology is an extension of ordinary topology that fuses two structures, the order and topological structures. Fuzzy interiors, boundaries, and exteriors play an important role in the uncertain relations between GIS objects. In this section we first present the basic definitions for fuzzy sets, and then the definitions for fuzzy mapping.

Definition 2.1 (Fuzzy subset). Let $X$ be a non-empty ordinary set and $I$ be the closed interval [0, $1]$.

An $I$-fuzzy subset on $X$ is a mapping $\mu_{A}: X \rightarrow I$, i.e., the family of all the [0,1]-fuzzy or $I$-fuzzy subsets on $X$ is just $I^{X}$; consisting of all the mappings from $X$ to $I$. Here, $I^{X}$ is called an $I$-fuzzy space. $X$ is called the carrier domain of each $I$-fuzzy subset in it, and $I$ is called the value domain of each $I$-fuzzy subset on $X . A \in I^{X}$ is called a crisp subset on $X$, if the image of the mapping is the subset of $\{0,1\} \subset I$.

Definition 2.2 (Rules of set relations). Let $A$ and $B$ be fuzzy sets in $X$ with membership functions $\mu_{A}(x)$ and $\mu_{B}(x)$, respectively. Then,

1) $A=B$, iff $\mu_{A}(x)=\mu_{B}(x)$ for all $x$ in $X$;

2) $A \leq B$, iff $\mu_{A}(x) \leq \mu_{B}(x)$ for all $x$ in $X$;

3) $C=A \vee B$, iff $\mu_{C}(x)=\max \left[\mu_{A}(x), \mu_{B}(x)\right]$ for all $x$ in $X$;

4) $D=A \wedge B$, iff $\mu_{D}(x)=\min \left[\mu_{A}(x), \mu_{B}(x)\right]$ for all $x$ in $X$;

5) $E=X \backslash A$, iff $\mu_{E}(x)=1-\mu_{A}(x)$ for all $x$ in $X$.

Definition 2.3 (Fuzzy topological space). Let $X$ be a non-empty ordinary set and $I=[0,1]$, Bol. Ciênc. Geod., sec. Artigos, Curitiba, v. 21, no 2, p.389-408, abr-jun, 2015. 
$\delta \subset I^{X} . \delta$ is called an $I$-fuzzy topology on $X$, and $\left(I^{X}, \delta\right)$ is called an $I$-fuzzy topological space (I-fts), if $\delta$ satisfies the following conditions:

1) $0,1 \in \delta$,2) If $A, B \in \delta$, then $A \wedge B \in \delta$, then 3) Let $\left\{A_{i}: i \in J\right\} \subset \delta$, where $\mathrm{J}$ is an index set, and $\vee_{i \in J} A_{i} \in \delta$.

Where, $0 \in \delta$ means the empty set and $1 \in \delta$ means the whole set $X$. The elements in $\delta$ are called open elements and the elements in the complement of $\delta$ are called closed elements, and the set of the complement of an open set is denoted by $\delta^{\prime}$.

Definition 2.4 (Interior and closure). For any fuzzy set $A \in I^{X}$, the interior of $A$ is defined as the join of all the open subsets contained in $A$, denoted by $A^{o}$. The closure of $\mathrm{A}$ is defined as the meeting of all the closed subsets containing A; denoted by $\bar{A}$.

Definition 2.5 (Fuzzy complement). For any fuzzy set $A$, we defined the complements of Aby $A^{C}(x)=1-A(x)$; denoted by $A^{C}$.

Definition 2.6 (Fuzzy boundary). The boundary of a fuzzy set $A$ is defined as: $\partial A=\bar{A} \wedge \overline{A^{C}}$.

Definition 2.7 (Closure operator). An operator $\alpha: I^{X} \rightarrow I^{X}$ is a fuzzy closure operator if the following conditions are satisfied:
1) $\alpha(0)=0$
2) $\alpha(A) \leq A$, for all $A \in I^{X}$;
3) $\alpha(A \vee B)=\alpha(A) \vee \alpha(B)$; 4) $\alpha(\alpha(A))=\alpha(A)$, for all $A \in I^{X}$.

Definition 2.8 (Interior operator). An operator $\alpha: I^{X} \rightarrow I^{X}$ is a fuzzy interior operator if the following conditions are satisfied:
1) $\alpha(1)=1$;
2) $A \leq \alpha(A)$, for all $A \in I^{X}$;
3) $\alpha(A \vee B)=\alpha(A) \wedge \alpha(B)$; 4) $\alpha(\alpha(A))=\alpha(A)$, for all $A \in I^{X}$.

Definition 2.9 (Interior and closure operators). For any fixed $\alpha \in[0,1]$, both operators, interior and closure, are defined as

$$
A_{\alpha}(x)=\left\{\begin{array}{ll}
A(x) & \text { if } \quad A(x)>\alpha \\
0 & \text { if } \quad A(x) \leq \alpha
\end{array} \text { and } A^{1-\alpha}(x)=\left\{\begin{array}{ll}
1 & \text { if } \quad A(x) \geq 1-\alpha \\
A(x) & \text { if } \quad A(x)<1-\alpha
\end{array}\right. \text { respectively, and }\right.
$$

can induce an $I$-fuzzy topology $\left(X, \tau_{\alpha}, \tau^{1-\alpha}\right)$ in $X$, where $\tau_{\alpha}=\left\{A_{\alpha}: A \in I^{X}\right\}$ are the open sets and $\tau^{1-\alpha}=\left\{A^{1-\alpha}: A \in I^{X}\right\}$ are the closed sets. The elements in $\tau_{\alpha}$ and $\tau^{1-\alpha}$ satisfy the relations $\left(A_{\alpha}\right)^{C}=\left(A^{C}\right)^{1-\alpha}$, for all fuzzy sets $A$, i.e., the complement of the elements in the $\tau_{\alpha}$ closed set. Details on how these two operators can induce a coherent $I$-fuzzy topology are Bol. Ciênc. Geod., sec. Artigos, Curitiba, v. 21, no 2, p.389-408, abr-jun, 2015. 
given in Liu and Shi (2006).

To study topological relations, it is essential to first understand the properties of fuzzy mapping, especially homeomorphic mapping since topological relations are invariant in homeomorphic mappings. The following section presents a number of definitions related to fuzzy mapping.

Let $I^{X}, I^{Y}$ be $I$-fuzzy spaces, $f: X \rightarrow Y$ an ordinary mapping. Based on $f: X \rightarrow Y$, define $I$-fuzzy mapping $f^{\rightarrow}: I^{X} \rightarrow I^{Y}$ and its $I$-fuzzy reverse mapping $f^{\rightarrow}: I^{X} \rightarrow I^{Y}$ by

$$
\begin{aligned}
& f^{\rightarrow}: I^{X} \rightarrow I^{Y}, f^{\rightarrow}(A)(y)=\left\{\begin{array}{ll}
V\{A(x)\}, \text { if } & x \in f^{-1}(y) \\
0 & \text { otherwise }
\end{array} \quad, \forall A \in I^{X}, \forall y \in Y\right. \\
& f^{\leftarrow}: I^{Y} \rightarrow I^{X}, f^{\leftarrow}(B)(x)=B(f(x)), \forall B \in I^{Y}, \forall x \in X
\end{aligned}
$$

While $I=[0,1]$.

Let $\left(I^{X}, \delta\right),\left(I^{Y}, \delta\right)$ be $I$-fts's, $f^{\rightarrow}:\left(I^{X}, \delta\right) \rightarrow\left(I^{Y}, \mu\right)$ is called $I$-fuzzy homeomorphism, if it is bijective, continuous, and open (Liu and Luo, 1997). One important theorem to check an I-fuzzy homeomorphism is that, as proved by Shi and Liu (2007). Let $A \in I^{X}, B \in I^{Y}$, let $\left(I^{X}, \delta\right),\left(I^{Y}, \delta\right)$ be $I$-fts's induced by an interior operator and closure operators. Then $f^{\rightarrow}:\left(I^{X}, \delta\right) \rightarrow\left(I^{Y}, \mu\right)$ is an $I$-fuzzy homeomorphism if and only if $f: X \rightarrow Y$ is a bijective mapping. Meanwhile, for the topology induced by these two operators, when checking a homeomorphic map, we only have to check whether there is a one-to-one correspondence between the domain and range.

\subsection{Fuzzy Simple Spatial Objects in GIS}

Based on the definitions presented in section 2.1, Liu and Shi developed fuzzy definitions for the basic elements in GIS (Liu and Shi, 2006, 2009; Shi and Liu, 2007), summarized here as follows:

Definition 2.10 (fuzzy point, Figure 1(a)). An $I$-fuzzy point on $X$ is an $I$-fuzzy subset $X_{\alpha} \in I^{X}$, defined as: $x_{\alpha}(y)=\left\{\begin{array}{ll}\alpha & \text { if } y=x \\ 0 & \text { otherwise }\end{array}\right.$.

Definition 2.11 (Simple fuzzy line, Figure 1(b)). A fuzzy subset in $X$ is called a simple fuzzy line $(L)$ if $L$ is a supported connected line in the background topology (i.e., a crisp line in the background topology).

Definition 2.12 (Simple fuzzy line segment, Figure 1(b)). The simple fuzzy line segment $(L)$ is a fuzzy subset in $X$ with: 1) for any $\alpha \in(0,1)$, the fuzzy line $L_{\alpha}$ is a supported connected line Bol. Ciênc. Geod., sec. Artigos, Curitiba, v. 21, no 2, p.389-408, abr-jun, 2015. 
segment (i.e., a crisp line segment in the background topology) in the background topology and 2) $\partial L\left(=\left(L_{\alpha}\right)^{C} \wedge L^{1-\alpha}\right)$ has at most two supported connected components.

Definition 2.13 (Simple fuzzy region, Figure 1(c)). A simple fuzzy region is a fuzzy region in $X$ where: 1) for any $\alpha \in(0,1)$, the fuzzy set $A_{\alpha}$ and $\partial A\left(A_{\alpha}\right)^{C} \wedge A^{1-\alpha}$ are two supported connected regular bounded open sets in the background topological space. And, 2) in the background topological space, any outward normal from Supp $\left(A_{\alpha}\right)$ must meet Supp $(\partial A)$ and have only one component.

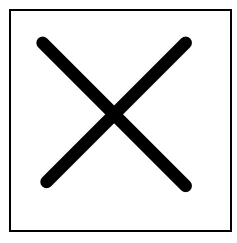

(a)Fuzzy point

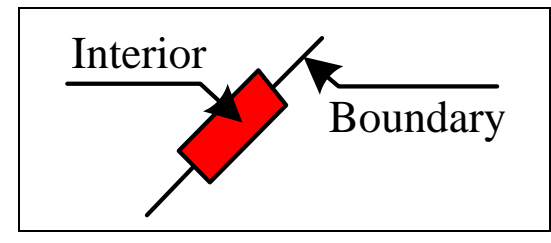

(b) Fuzzy line segment

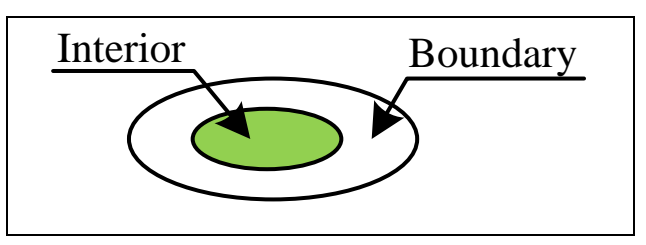

(c) Fuzzy region

Figure1: (a) Fuzzy point for a given $\alpha$; (b) Fuzzy line segment for a given $\alpha$; (c) Fuzzy region for a given $\alpha$ (Liu and Shi 2009)

On the basis of definitions for simple fuzzy points, line segments and regions, Shi and Liu (2007) provide an example of computing the interior, boundary, and exterior of spatial objects for different $\alpha$ values, and the interior, boundary, and exterior of spatial objects were confirmed for each given $\alpha$ value. Based on the fuzzy definitions, Liu and Shi (2009) proposed a new $3 \times 3$ integration model to compute the topological relations between simple fuzzy region, line segment and fuzzy points. The element $\left(f_{X} A \wedge B d V\right)$ of the new $3 \times 3$ integration model is the ratio of the area( or volume) of the meet of two fuzzy spatial objects in a join of two simple spatial object(here a join of two fuzzy objects means "union" of two fuzzy objects; a meet of two fuzzy objects means "intersection" of two fuzzy objects(Liu and Shi, 2009)). And it was difficult to change or transform the new $3 \times 3$ integration model to describe the topological relations between one simple crisp spatial object and one simple fuzzy spatial object.

Based on existing related studies, in the next section, we will discuss the method of fuzzy topological relations for simple spatial objects.

\section{Modeling Fuzzy Topological Relations for Simple Spatial Objects in GIS}

\subsection{A New Definition for Simple Fuzzy Spatial Objects}

Based on section 2, we developed a new definition for a simple fuzzy spatial line segment and region by applying the definition presented in this section. On fuzzy topological space (Chang, 
1968), the fuzzy point definition remains the same as Definition 2.10.

Definition 2.14 (inner and outer boundary of simple fuzzy line segment, Figure 2(a)), for given $\alpha$, the interior and boundary of simple line segment $L($ as shown in figurel $(b))$ are confirmed, separately, can be regarded as a simple crisp line segment. Therefore, we define $\partial L_{\alpha}^{-}$as the outer-boundary of $L$, and $\partial L_{\alpha}^{o}$ as the inner-boundary of $L, L_{\alpha}^{o}$ as the interior of $L$, and $\partial L_{\alpha}$ as the boundary of $L($ as shown in figure $4(a)): \partial L_{\alpha}=\partial L_{\alpha}^{-}-\partial L_{\alpha}^{o}$. So, a simple fuzzy line segment $L$ for given $\alpha$ can be written as: $L_{\alpha}=\partial L_{\alpha}^{-} \cup \partial L_{\alpha} \cup \partial L_{\alpha}^{o} \cup L_{\alpha}^{o}$.

Definition 2.15 (inner and outer boundary of simple fuzzy region, Figure 2(b)), for a given $\alpha$, the interior and boundary of fuzzy region $A$ (as shown in figurel(c)) is confirmed, separately, can be regarded as a simple crisp region. Therefore, we defined $\partial A_{\alpha}^{-}$as the outer-boundary of $A$, and $\partial A_{\alpha}^{o}$ as the inner-boundary of $A, A_{\alpha}^{o}$ as the interior of $A$ and $\partial A_{\alpha}$ as the boundary of $A$ (as shown in figure $4(b)): \quad \partial A_{\alpha}=\partial A_{\alpha}^{-}-\partial A_{\alpha}^{o}$. So the fuzzy region $A$ for given $\alpha$ can be expressed as: $A_{\alpha}=\partial A_{\alpha}^{-} \cup \partial A_{\alpha} \cup \partial A_{\alpha}^{o} \cup A_{\alpha}^{o}$. Meanwhile, $\partial A_{\alpha}^{-}$and $\partial A_{\alpha}^{o}$ can be considered as two simple crisp regions.

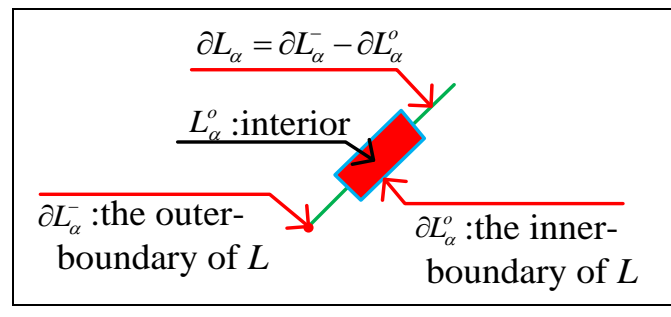

(a) Fuzzy line segment $L$

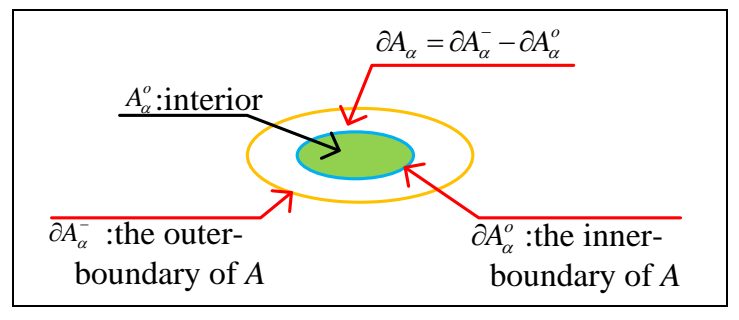

(b) Fuzzy region $A$

Figure 2: (a) The inner-outer boundary of simple fuzzy line segment $L$ for given $\alpha$; (b) The inner-outer boundary of simple fuzzy region $A$ for given $\alpha$

Based on the above definitions, (1) to a simple fuzzy line segment $L$ for given $\alpha$, if $\partial L_{\alpha}^{-}=\partial L_{\alpha}^{o}$, that is $\partial L_{\alpha}=0$, and $L=L_{\alpha}=\partial L_{\alpha}^{-} \cup L_{\alpha}^{o}$, it means $L$ is a simple crisp line segment; (2) to a fuzzy region $A$ for given $\alpha$, if $\partial A_{\alpha}^{-}=\partial A_{\alpha}^{o}$, that is $\partial A_{\alpha}=0, A=A_{\alpha}=\partial A_{\alpha}^{-} \cup A_{\alpha}^{o}$, it means $A$ is a simple crisp region.

Based on these definitions, the next section will primarily focus on discussing the new model of fuzzy topological relations for simple spatial objects. 


\subsection{A New Model of the Fuzzy Topological Relations for Simple Spatial}

\section{Objects}

In this paper, we just discussed the topological relations between two simple fuzzy line segments, the topological relations one simple fuzzy line segment and one simple fuzzy region, and the topological relations between two simple fuzzy regions, as follows.

\section{(I) Topological relations between two simple fuzzy line segments}

For one simple fuzzy line segment $L 1$ for given $\alpha$ (figure3 (a)), and the other simple fuzzy line segment $L 2$ for given $\beta$ (figure3 (b)). The topological relations between $L 1$ and $L 2$ can be computed by $4 \times 4$ intersection model as equation (1).

$$
R\left(L 1_{\alpha}, L 2_{\beta}\right)=\left[\begin{array}{llll}
\partial L 1_{\alpha}^{-} \cap \partial L 2_{\beta}^{-} & \partial L 1_{\alpha}^{-} \cap \partial L 2_{\beta} & \partial L 1_{\alpha}^{-} \cap \partial L 2_{\beta}^{o} & \partial L 1_{\alpha}^{-} \cap L 2_{\beta}^{o} \\
\partial L 1_{\alpha} \cap \partial L 2_{\beta}^{-} & \partial L 1_{\alpha} \cap \partial L 2_{\beta} & \partial L 1_{\alpha} \cap \partial L 2_{\beta}^{o} & \partial L 1_{\alpha} \cap L 2_{\beta}^{o} \\
\partial L 1_{\alpha}^{o} \cap \partial L 2_{\beta}^{-} & \partial L 1_{\alpha}^{o} \cap \partial L 2_{\beta} & \partial L 1_{\alpha}^{o} \cap \partial L 2_{\beta}^{o} & \partial L 1_{\alpha}^{o} \cap L 2_{\beta}^{o} \\
L 1_{\alpha}^{o} \cap \partial L 2_{\beta}^{-} & L 1_{\alpha}^{o} \cap \partial L 2_{\beta} & L 1_{\alpha}^{o} \cap \partial L 2_{\beta}^{o} & L 1_{\alpha}^{o} \cap L 2_{\beta}^{o}
\end{array}\right]
$$

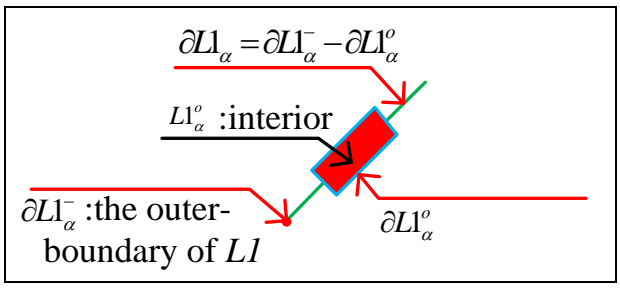

(a)

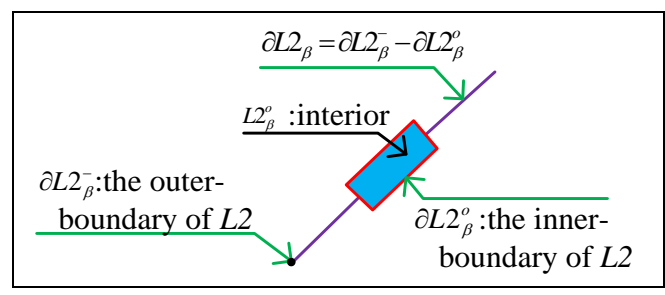

(b)

Figure 3: (a) A simple fuzzy line segment $L 1$ for given $\alpha$; (b) A simple fuzzy line segment $L 2$ for given $\beta$

There are three different topological relations between $L 1$ and $L 2$, as follows:

1) if $\partial L 1_{\alpha}^{-}=\partial L 1_{\alpha}^{o}$,that is $\partial L 1_{\alpha}=0, L 1=L 1_{\alpha}=\partial L 1_{\alpha}^{-} \cup L 1_{\alpha}^{o} ; \quad$ if $\partial L 2_{\beta}^{-}=\partial L 2_{\beta}^{o}$, that is $\partial L 2_{\beta}=0, L 2=L 2_{\beta}=\partial L 2_{\beta}^{-} \cup L 2_{\beta}^{o}$. Thus, $L 1$ and $L 2$ are crisp line segments, the equation (1) can be turned into 4-Intersection Model (4IM) (Egenhofer and Franzosa, 1991a) or 9-Intersection Model (9IM) (Egenhofer and Franzosa, 1991b), the topological relations between $L 1$ and $L 2$ are computed by 4IM or 9IM.

2) If $\partial L 1_{\alpha}^{-}=\partial L 1_{\alpha}^{o}$, that is $\partial L 1_{\alpha}=0, L 1=L 1_{\alpha}=\partial L 1_{\alpha}^{-} \cup L 1_{\alpha}^{o}, L 1$ is a simple crisp line segment. If $\partial L 2_{\beta}^{-} \neq \partial L 2_{\beta}^{o}$, that is $\partial L 2_{\beta} \neq 0, L 2=L 2_{\beta}=\partial L 2_{\beta}^{-} \cup \partial L 2_{\beta} \cup \partial L 2_{\beta}^{o} \cup L 2_{\beta}^{o}, L 2$ is a simple fuzzy line segment, and comprised of four components $\partial L 2_{\beta}^{-}, \partial L 2_{\beta}, \partial L 2_{\beta}^{o}$ and $L 2_{\beta}^{o}$, 
while $\partial L 2_{\beta}=\partial L 2_{\beta}^{-}-\partial L 2_{\beta}^{o}$. The equation (1) can be turned into $2 \times 4$ intersection model as equation (2).

$$
R\left(L 1_{\alpha}, L 2_{\beta}\right)=\left[\begin{array}{llll}
\partial L 1_{\alpha} \cap \partial L 2_{\beta}^{-} & \partial L 1_{\alpha} \cap \partial L 2_{\beta} & \partial L 1_{\alpha} \cap \partial L 2_{\beta}^{o} & \partial L 1_{\alpha} \cap L 2_{\beta}^{o} \\
L 1_{\alpha}^{o} \cap \partial L 2_{\beta}^{-} & L 1_{\alpha}^{o} \cap \partial L 2_{\beta} & L 1_{\alpha}^{o} \cap \partial L 2_{\beta}^{o} & L 1_{\alpha}^{o} \cap L 2_{\beta}^{o}
\end{array}\right]
$$

3) If $\partial L 1_{\alpha}^{-} \neq \partial L 1_{\alpha}^{o}$, that is $\partial L 1_{\alpha} \neq 0, L 1=L 1_{\alpha}=\partial L 1_{\alpha}^{-} \cup \partial L 1_{\alpha} \cup \partial L 1_{\alpha}^{o} \cup L 1_{\alpha}^{o}, L 1$ is a simple fuzzy line segment, and comprised of four components $\partial L 1_{\alpha}^{-}, \partial L 1_{\alpha}, \partial L 1_{\alpha}^{o}$ and $L 1_{\alpha}^{o}$, in addition, $\quad \partial L 1_{\alpha}=\partial L 1_{\alpha}^{-}-\partial L 1_{\alpha}^{o}$. If $\partial L 2_{\beta}^{-} \neq \partial L 2_{\beta}^{o}$, that is $\partial L 2_{\beta} \neq 0$, $L 2=L 2_{\beta}=\partial L 2_{\beta}^{-} \cup \partial L 2_{\beta} \cup \partial L 2_{\beta}^{o} \cup L 2_{\beta}^{o}, L 2$ is a simple fuzzy line segment too, comprised of four components $\partial L 2_{\beta}^{-}, \partial L 2_{\beta}, \partial L 2_{\beta}^{o}$ and $L 2_{\beta}^{o}$, in addition, $\partial L 2_{\beta}=\partial L 2_{\beta}^{-}-\partial L 2_{\beta}^{o}$. Thus, the topological relations between $L 1$ and $L 2$ can be computed by $4 \times 4$ intersection model as equation (1).

\section{(II)Topological relations between one simple fuzzy line segment and one simple fuzzy region}

For one simple fuzzy line segment $L 1$ for a given $\alpha$ (figure4 (a)), there is one simple fuzzy region $A l$ for given $\beta$ (figure4 (b)). The topological relations between $L 1$ and $A l$ can be computed by $4 \times 4$ intersection model as equation (3).

$$
R\left(L_{\alpha}, A_{\beta}\right)=\left[\begin{array}{llll}
\partial L_{\alpha}^{-} \cap \partial A_{\beta}^{-} & \partial L_{\alpha}^{-} \cap \partial A_{\beta} & \partial L_{\alpha}^{-} \cap \partial A_{\beta}^{o} & \partial L_{\alpha}^{-} \cap A_{\beta}^{o} \\
\partial L_{\alpha} \cap \partial A_{\beta}^{-} & \partial L_{\alpha} \cap \partial A_{\beta} & \partial L_{\alpha} \cap \partial A_{\beta}^{o} & \partial L_{\alpha} \cap A_{\beta}^{o} \\
\partial L_{\alpha}^{o} \cap \partial A_{\beta}^{-} & \partial L_{\alpha}^{o} \cap \partial A_{\beta} & \partial L_{\alpha}^{o} \cap \partial A_{\beta}^{o} & \partial L_{\alpha}^{o} \cap A_{\beta}^{o} \\
L_{\alpha}^{o} \cap \partial A_{\beta}^{-} & L_{\alpha}^{o} \cap \partial A_{\beta} & L_{\alpha}^{o} \cap \partial A_{\beta}^{o} & L_{\alpha}^{o} \cap A_{\beta}^{o}
\end{array}\right]
$$

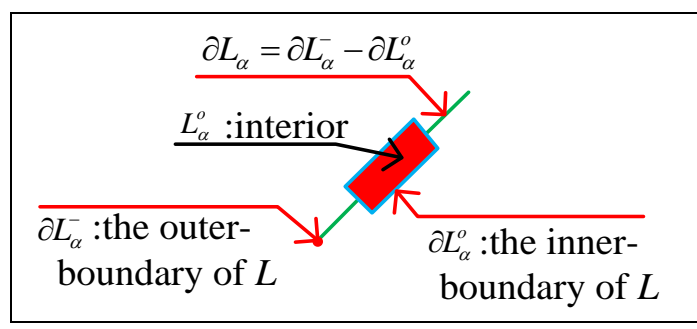

(a)

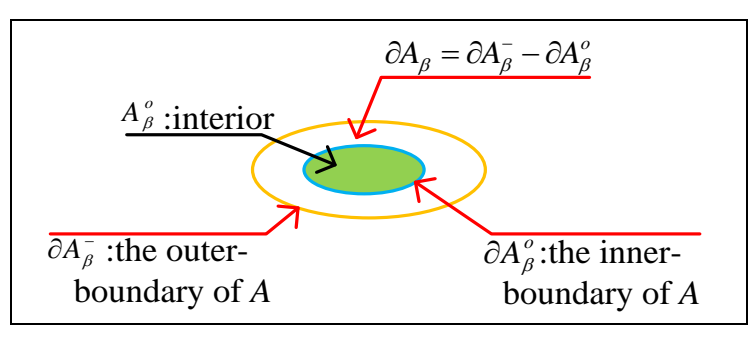

(b)

Figure 4: (a) A simple fuzzy line segment $L$ for given $\alpha$; (b)A simple fuzzy region $A$ for given $\beta$

There are four different topological relations between $L 1$ and $A 1$, as follows:

1) If $\partial L_{\alpha}^{-}=\partial L_{\alpha}^{o}$, that is $\partial L_{\alpha}=0, L=L_{\alpha}=\partial L_{\alpha}^{-} \cup L_{\alpha}^{o}, L$ is a simple crisp line segment. If $\partial A_{\beta}^{-}=\partial A_{\beta}^{o}$, 
that is $\partial A_{\beta}=0, A=A_{\beta}=\partial A_{\beta}^{-} \cup A_{\beta}^{o}$, and $A$ is a simple crisp region. The equation (3) can be turned into 4-Intersection Model (4IM) (Egenhofer and Franzosa, 1991a) or 9-Intersection Model (9IM) (Egenhofer and Franzosa, 1991b), the topological relations between $L$ and $A$ are computed by 4IM or 9IM.

2) If $\partial L_{\alpha}^{-}=\partial L_{\alpha}^{o}$, that is $\partial L_{\alpha}=0, L=L_{\alpha}=\partial L_{\alpha}^{-} \cup L_{\alpha}^{o}, L$ is a simple crisp line segment. If $\partial A_{\beta}^{-} \neq \partial A_{\beta}^{o}$, that is $\partial A_{\beta} \neq 0, A=A_{\beta}=\partial A_{\beta}^{-} \cup \partial A_{\beta} \cup \partial A_{\beta}^{o} \cup A_{\beta}^{o}, A$ is a simple fuzzy region, comprised of four components $\partial A_{\beta}^{-}, \partial A_{\beta}, \partial A_{\beta}^{o}$ and $A_{\beta}^{o}$, in addition, $\partial A_{\beta}=\partial A_{\beta}^{-}-\partial A_{\beta}^{o}$. The equation (3) can be turned into $2 \times 4$ intersection model as equation (4).

$$
R\left(L_{\alpha}, A_{\beta}\right)=\left[\begin{array}{llll}
\partial L_{\alpha} \cap \partial A_{\beta}^{-} & \partial L_{\alpha} \cap \partial A_{\beta} & \partial L_{\alpha} \cap \partial A_{\beta}^{o} & \partial L_{\alpha} \cap A_{\beta}^{o} \\
L_{\alpha}^{o} \cap \partial A_{\beta}^{-} & L_{\alpha}^{o} \cap \partial A_{\beta} & L_{\alpha}^{o} \cap \partial A_{\beta}^{o} & L_{\alpha}^{o} \cap A_{\beta}^{o}
\end{array}\right]
$$

3) If $\partial L_{\alpha}^{-} \neq \partial L_{\alpha}^{o}$, that is $\partial L_{\alpha} \neq 0, L=L_{\alpha}=\partial L_{\alpha}^{-} \cup \partial L_{\alpha} \cup \partial L_{\alpha}^{o} \cup L_{\alpha}^{o}, L$ is a simple fuzzy line segment, comprised of four components $\partial L_{\alpha}^{-}, \partial L_{\alpha}, \partial L_{\alpha}^{o}$ and $L_{\alpha}^{o}$, in addition, $\partial L_{\alpha}=\partial L_{\alpha}^{-}-\partial L_{\alpha}^{o}$.If $\partial A_{\beta}^{-}=\partial A_{\beta}^{o}$, that is $\partial A_{\beta}=0, A=A_{\beta}=\partial A_{\beta}^{-} \cup A_{\beta}^{o}, A$ is a simple crisp region. The equation (3) can be turned into $2 \times 4$ intersection model too, as equation (5).

$$
R\left(L_{\alpha}, A_{\beta}\right)=\left[\begin{array}{cccc}
\partial A_{\beta} \cap \partial L_{\alpha}^{-} & \partial A_{\beta} \cap \partial L_{\alpha} & \partial A_{\beta} \cap \partial L_{\alpha}^{o} & \partial A_{\beta} \cap L_{\alpha}^{o} \\
A_{\beta}^{o} \cap \partial L_{\alpha}^{-} & A_{\beta}^{o} \cap \partial L_{\alpha} & A_{\beta}^{o} \cap \partial L_{\alpha}^{o} & A_{\beta}^{o} \cap L_{\alpha}^{o}
\end{array}\right]
$$

4) If $\partial L_{\alpha}^{-} \neq \partial L_{\alpha}^{o}$, that is $\partial L_{\alpha} \neq 0, L=L_{\alpha}=\partial L_{\alpha}^{-} \cup \partial L_{\alpha} \cup \partial L_{\alpha}^{o} \cup L_{\alpha}^{o}, L$ is a simple fuzzy line segment, comprised of four components $\partial L_{\alpha}^{-}, \partial L_{\alpha}, \partial L_{\alpha}^{o}$ and $L_{\alpha}^{o}$, in addition, $\partial L_{\alpha}=\partial L_{\alpha}^{-}-\partial L_{\alpha}^{o}$.If $\partial A_{\beta}^{-} \neq \partial A_{\beta}^{o}$, that is $\partial A_{\beta} \neq 0, A=A_{\beta}=\partial A_{\beta}^{-} \cup \partial A_{\beta} \cup \partial A_{\beta}^{o} \cup A_{\beta}^{o}, A$ is a simple fuzzy region, comprised of four components $\partial A_{\beta}^{-}, \partial A_{\beta}, \partial A_{\beta}^{o}$ and $A_{\beta}^{o}$, in addition, $\partial A_{\beta}=\partial A_{\beta}^{-}-\partial A_{\beta}^{o}$. The topological relations between $L$ and $A$ can be computed by $4 \times 4$ intersection model as equation (3).

\section{(III) Topological relations between two simple fuzzy regions}

For one simple fuzzy region $A 1$ for given $\alpha$ (figure5 (a)), the other simple fuzzy region $A 2$ for given $\beta$ (figure5 (b)). The topological relations between $A 1$ and $A 2$ can be computed by $4 \times 4$ intersection model as equation (6). 
$R\left(A 1_{\alpha}, A 2_{\beta}\right)=\left[\begin{array}{llll}\partial A 1_{\alpha}^{-} \cap \partial A 2_{\beta}^{-} & \partial A 1_{\alpha}^{-} \cap \partial A 2_{\beta} & \partial A 1_{\alpha}^{-} \cap \partial A 2_{\beta}^{o} & \partial A 1_{\alpha}^{-} \cap A 2_{\beta}^{o} \\ \partial A 1_{\alpha} \cap \partial A 2_{\beta}^{-} & \partial A 1_{\alpha} \cap \partial A 2_{\beta} & \partial A 1_{\alpha} \cap \partial A 2_{\beta}^{o} & \partial A 1_{\alpha} \cap A 2_{\beta}^{o} \\ \partial A 1_{\alpha}^{o} \cap \partial A 2_{\beta}^{-} & \partial A 1_{\alpha}^{o} \cap \partial A 2_{\beta} & \partial A 1_{\alpha}^{o} \cap \partial A 2_{\beta}^{o} & \partial A 1_{\alpha}^{o} \cap A 2_{\beta}^{o} \\ A 1_{\alpha}^{o} \cap \partial A 2_{\beta}^{-} & A 1_{\alpha}^{o} \cap \partial A 2_{\beta} & A 1_{\alpha}^{o} \cap \partial A 2_{\beta}^{o} & A 1_{\alpha}^{o} \cap A 2_{\beta}^{o}\end{array}\right]$

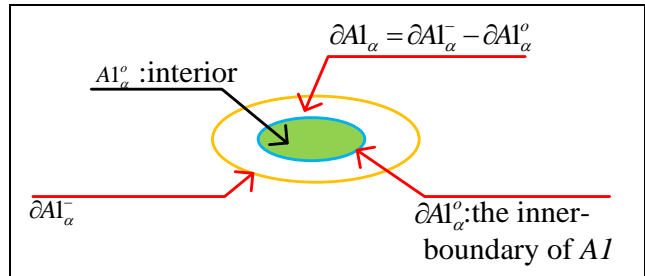

(a)

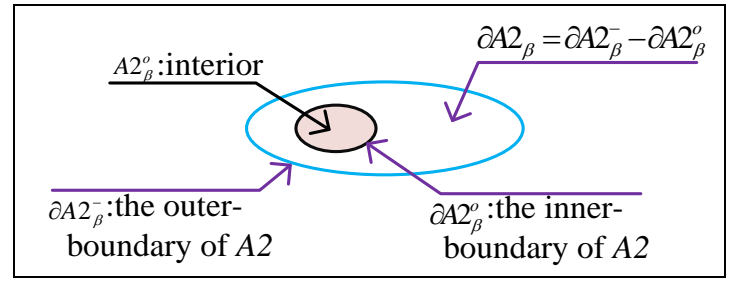

(b)

Figure 5: (a) A simple fuzzy region $A 1$ for given $\alpha$; (b) A simple fuzzy region $A 2$ for given $\beta$ There are three different topological relations between $A 1$ and $A 2$, as follows:

1) if $\partial A 1_{\alpha}^{-}=\partial A 1_{\alpha}^{o}$, that is $\partial A 1_{\alpha}=0, A 1=A 1_{\alpha}=\partial A 1_{\alpha}^{-} \cup A 1_{\alpha}^{o}, A 1$ is a simple crisp region. If $\partial A 2_{\beta}^{-}=\partial A 2_{\beta}^{o}$, that is $\partial A 2_{\beta}=0, A 2=A 2_{\beta}=\partial A 2_{\beta}^{-} \cup A 2_{\beta}^{o}, A 2$ is a simple crisp region too. Therefore, the equation (6) can be turned into 4-Intersection Model (4IM) (Egenhofer and Franzosa, 1991a) or 9-Intersection Model (9IM) (Egenhofer and Franzosa, 1991b), the topological relations between $A 1$ and $A 2$ are computed by 4IM or 9IM.

2) If $\partial A 1_{\alpha}^{-}=\partial A 1_{\alpha}^{o}$, that is $\partial A 1_{\alpha}=0, A 1=A 1_{\alpha}=\partial A 1_{\alpha}^{-} \cup A 1_{\alpha}^{o}, A 1$ is a simple crisp region. If $\partial A 2_{\beta}^{-} \neq \partial A 2_{\beta}^{o}$, that is $\partial A 2_{\beta} \neq 0, A 2=A 2_{\beta}=\partial A 2_{\beta}^{-} \cup \partial A 2_{\beta} \cup \partial A 2_{\beta}^{o} \cup A 2_{\beta}^{o}, A 2$ is a simple fuzzy region, comprised of four components $\partial A 2_{\beta}^{-}, \partial A 2_{\beta}, \partial A 2_{\beta}^{o}$ and $A 2_{\beta}^{o}, \quad$ in addition, $\partial A 2_{\beta}=\partial A 2_{\beta}^{-}-\partial A 2_{\beta}^{o}$. The equation (6) can be turned into $2 \times 4$ intersection model as equation(7).

$$
R\left(A 1_{\alpha}, A 2_{\beta}\right)=\left[\begin{array}{llll}
\partial A 1_{\alpha} \cap \partial A 2_{\beta}^{-} & \partial A 1_{\alpha} \cap \partial A 2_{\beta} & \partial A 1_{\alpha} \cap \partial A 2_{\beta}^{o} & \partial A 1_{\alpha} \cap A 2_{\beta}^{o} \\
A 1_{\alpha}^{o} \cap \partial A 2_{\beta}^{-} & A 1_{\alpha}^{o} \cap \partial A 2_{\beta} & A 1_{\alpha}^{o} \cap \partial A 2_{\beta}^{o} & A 1_{\alpha}^{o} \cap A 2_{\beta}^{o}
\end{array}\right]
$$

3) If $\partial A 1_{\alpha}^{-} \neq \partial A 1_{\alpha}^{o}$, that is $\partial A 1_{\alpha} \neq 0$, and $A 1=A 1_{\alpha}=\partial A 1_{\alpha}^{-} \cup \partial A 1_{\alpha} \cup \partial A 1_{\alpha}^{o} \cup A 1_{\alpha}^{o}, A 1$ is a simple fuzzy region, comprised of four components $\partial A 1_{\alpha}^{-}, \partial A 1_{\alpha}, \partial A 1_{\alpha}^{o}$ and $A 1_{\alpha}^{o}$, in addition,

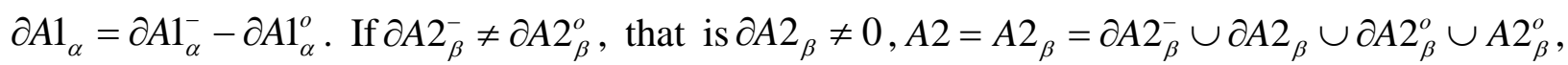
$A 2$ is a simple fuzzy region, comprised of four components $\partial A 2_{\beta}^{-}, \partial A 2_{\beta}, \partial A 2_{\beta}^{o}$ and $A 2_{\beta}^{o}$, in addition, $\partial A 2_{\beta}=\partial A 2_{\beta}^{-}-\partial A 2_{\beta}^{o}$. The topological relations between $A 1$ and $A 2$ can be computed Bol. Ciênc. Geod., sec. Artigos, Curitiba, v. 21, no 2, p.389-408, abr-jun, 2015. 
by $4 \times 4$ intersection model as equation (6).

Through the above description, the equation(1), (3) and (6)are equivalent, only replacing the elements of the $4 \times 4$ intersection model, the $4 \mathrm{IM}$ or $9 \mathrm{IM}$, equation(2), (4) ,(5), (7) are only the new $4 \times 4$ intersection models'(equation(1), (3), (6)) exception, and all the equation can describe the topological relations respectively.

In this section, we develop a new $4 \times 4$ intersection model to compute the fuzzy topological relations between simple spatial objects. An analysis of the new model exposes: (1) the topological relations between two simple crisp line segments; (2) the topological relations between two simple fuzzy line segments; (3) the topological relations between one simple crisp line segment and one simple fuzzy line segment; (4) the topological relations between one simple crisp line segment and one simple crisp region; (5) the topological relations between one simple crisp line segment and one simple fuzzy region; (6) the topological relations between one simple fuzzy line segment and one simple crisp region; (7) the topological relations between one simple fuzzy line segment and one simple fuzzy region; (8) the topological relations between one simple crisp region and one simple fuzzy region; (9) the topological relations between two simple crisp regions; (10) the topological relations between two simple fuzzy regions.

In the next section, we will focus on taking some examples to demonstrate the validity of the new model by comparing with existing models.

\section{Experiment and Comparison}

The new $4 \times 4$ intersection model can identify the topological relations between two simple spatial objects. The following content will take some examples to demonstrate the validity of the new model, and compare with the existing fuzzy models.

\subsection{Experiment Results}

In this section, we will take some examples to demonstrate the validity of the new model.

1) A simple crisp line segment $L 1$ (figure6 (a)), a simple fuzzy line segment $L 2$ for given $\beta=0.4$ (figure6 (b)).The topological relation between them was shown in figure 6(c). Since the intersection of two sets can be either 0 or 1, the topological relation between $L 1$ and $L 2$ can be computed by equation (2) as: $R(L 1, L 2)=\left[\begin{array}{llll}0 & 0 & 0 & 0 \\ 0 & 0 & 1 & 1\end{array}\right]$. 


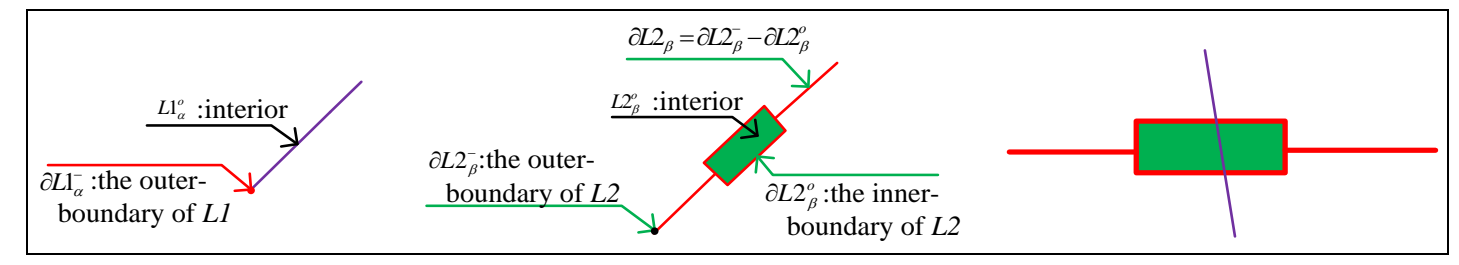

(a)

(b)

(c)

Figure 6: (a) Simple crisp line segment $L 1$; (b) Simple fuzzy line segment $L 2$ for given $\beta=0.4$;

(c) The topological relation between $L 1$ and $L 2$

2) A simple fuzzy line segment $L 1$ for given $\alpha=0.5$ (figure7 (a)), a simple fuzzy line segment $L 2$ for given $\beta=0.3$ (figure7 (b)).The topological relation between them was shown in figure7(c).Since the intersection of two sets can be either 0 or 1 , the topological relation between $L 1$ and $L 2$ can be computed by equation (1) as:

$$
R(L 1, L 2)=\left[\begin{array}{llll}
0 & 1 & 0 & 1 \\
0 & 1 & 1 & 1 \\
0 & 1 & 0 & 0 \\
0 & 1 & 0 & 0
\end{array}\right]
$$

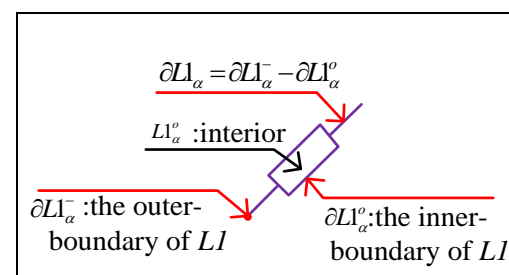

(a)

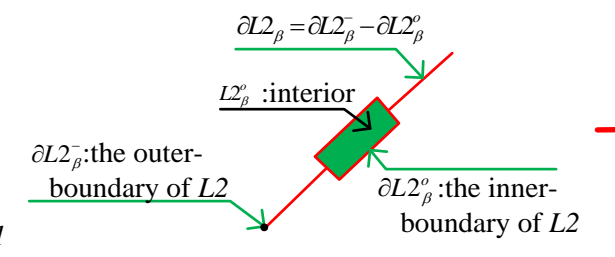

(b)

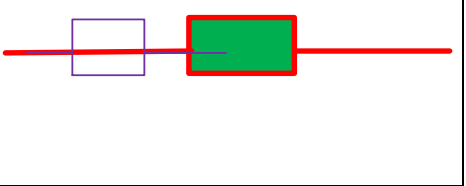

(c)

Figure 7: (a) Simple fuzzy line segment $L 1$ for given $\alpha=0.5$; (b) Simple fuzzy line segment $L 2$ for given $\beta=0.3$; (c) The topological relation between $L 1$ and $L 2$

3) A simple fuzzy line segment $L 1$ for given $\alpha=0.5$ (figure8 (a)), a simple fuzzy region $A 1$ for given $\beta=0.4$ (figure8 (b)). The topological relation between them was shown in figure8(c). Since the intersection of two sets can be either 0 or 1 , the topological relation between $L 1$ and $A l$ can be computed by equation (3) as:

$$
R(L 1, A 1)=\left[\begin{array}{cccc}
0 & 0 & 0 & 1 \\
0 & 1 & 1 & 1 \\
1 & 1 & 0 & 0 \\
1 & 1 & 0 & 0
\end{array}\right]
$$




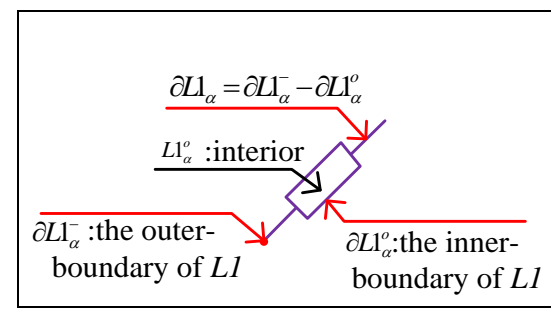

(a)

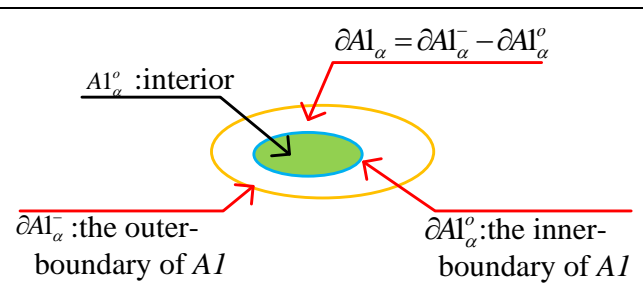

(b)

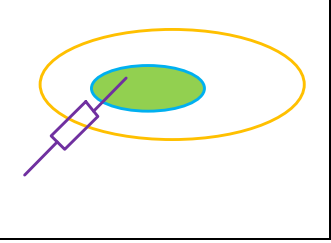

(c)

Figure 8: (a) Simple fuzzy line segment $L 1$ for given $\alpha=0.5$; (b) Simple fuzzy region $A 1$ for given $\beta=0.4$; (c) The topological relation between $L 1$ and $A 1$

4) A simple crisp line segment $L 1$ (figure9 (a)), a simple fuzzy region $A 1$ for given $\beta=0.4$ (figure 9(b)). The topological relation between them was shown in figure9(c). Since the intersection of two sets can be either 0 or 1 , the topological relation between $L 1$ and $A l$ can be computed by equation (4) as: $R(L 1, A 1)=\left[\begin{array}{cccc}0 & 0 & 0 & 1 \\ 1 & 1 & 1 & 1\end{array}\right]$

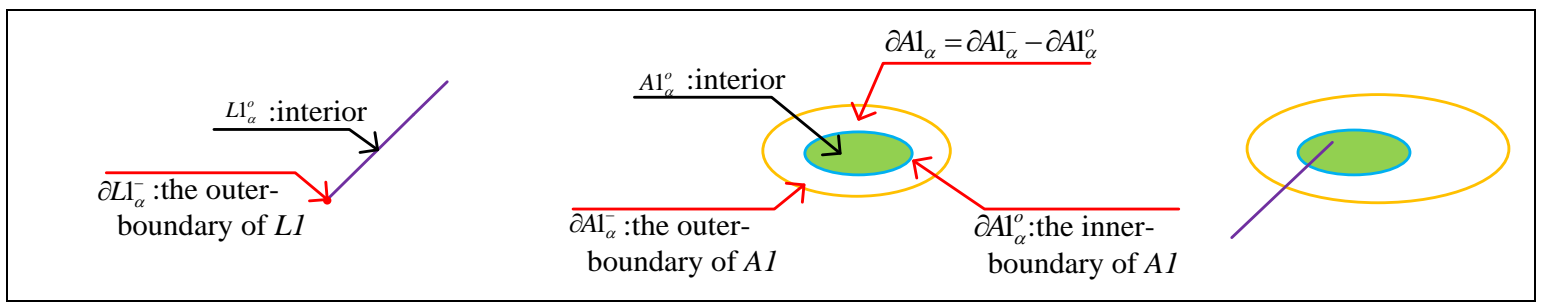

(a)

(b)

(c)

Figure 9: (a) Simple crisp line segment $L 1$; (b) Simple fuzzy region $A 1$ for given $\beta=0.4$; (c) The topological relation between $L 1$ and $A 1$

5) A simple fuzzy line segment $L 1$ for given $\alpha=0.5$ (figure10 (a)), a simple crisp region $A 1$ (figure10 (b)). The topological relation between them was shown in figure10(c). Since the intersection of two sets can be either 0 or 1, the topological relation between $L 1$ and $A l$ can be computed by equation (5) as: $R(L 1, A 1)=\left[\begin{array}{cccc}0 & 0 & 1 & 1 \\ 1 & 1 & 1 & 1\end{array}\right]$.

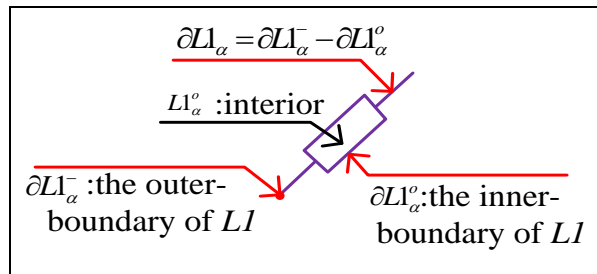

(a)

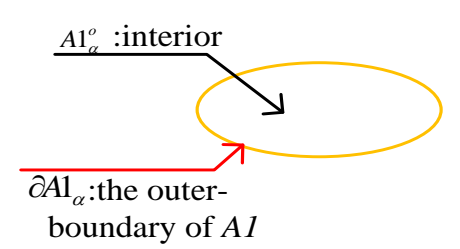

(b)

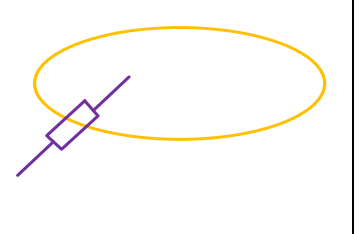

(c)

Figure 10: (a) Simple fuzzy line segment $L 1$ for given $\alpha=0.5$; (b) Simple crisp region $A 1$; (c)

The topological relation between $L 1$ and $A l$

6) A simple fuzzy region $A 1$ for given $\alpha=0.5$ (figure11 (a)), a simple fuzzy region $A 2$ for given $\beta=0.3$ (figure11 (b)), the topological relation between them was shown in figure11(c). Bol. Ciênc. Geod., sec. Artigos, Curitiba, v. 21, no 2, p.389-408, abr-jun, 2015. 
Since the intersection of two sets can be either 0 or 1 , the topological relation between $A 1$ and $A 2$

can be computed by equation (6) as: $R(A 1, A 2)=\left[\begin{array}{llll}1 & 1 & 1 & 1 \\ 1 & 1 & 1 & 1 \\ 1 & 1 & 0 & 0 \\ 1 & 1 & 0 & 0\end{array}\right]$.

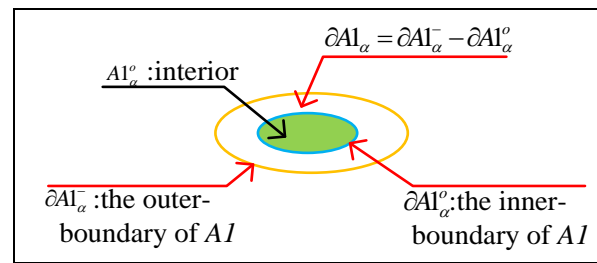

(a)

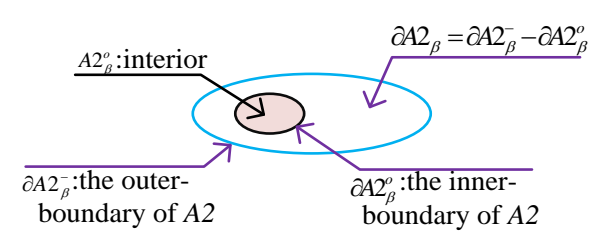

(b)

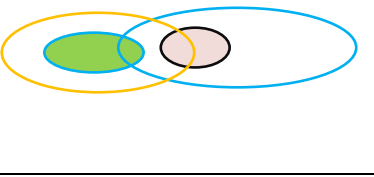

(c)

Figure 11: (a) Simple fuzzyregion $A$ lfor given $\alpha=0.5$; (b) Simple fuzzy region $A 2$ for given $\beta=0.3$; (c) The topological relation between $A$ land $A 2$

7) A simple fuzzy region $A 1$ for given $\alpha=0.5$ (figure12 (a)), a simple crisp region $A 2$ (figure 12 (b)), the topological relation between them was shown in figure12(c). Since the intersection of two sets can be either 0 or 1, the topological relation between $A 1$ and $A 2$ can be computed by equation (7) as: $R(A 1, A 2)=\left[\begin{array}{llll}1 & 1 & 1 & 1 \\ 1 & 1 & 1 & 1\end{array}\right]$.

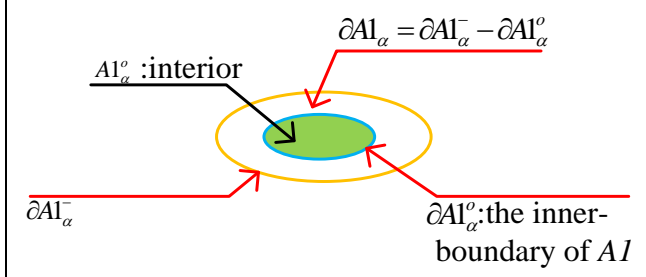

(a)

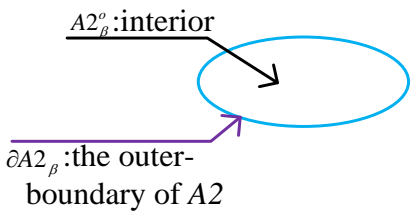

(b)

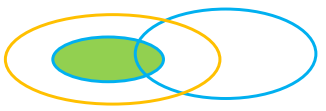

(c)

Figure 12: (a) Simple fuzzyregion $A 1$ for given $\alpha=0.5$; (b) Simple crisp region $A 2$; (c) the topological relation between $A 1$ and $A 2$

\subsection{Comparison with Existing Models}

In dealing with fuzzy spatial objects, Cohn and Gotts (1996) proposed the 'egg-yolk' model with two concentric sub-regions, indicating the degree of 'membership' in a vague/fuzzy region. In this model, the 'yolk' represents the precise part and 'egg' represents the vague/fuzzy part of a region. The 'egg-yolk' model is an extension of RCC theory into the vague/fuzzy region. A total of 46 relations can be identified (Cohn and Gotts, 1996). Based on the 9-intersection 
model $R_{9}(A, B)=\left[\begin{array}{lll}A^{\circ} \cap B^{\circ} & A^{\circ} \cap \partial B & A^{\circ} \cap B^{-} \\ \partial A \cap B^{\circ} & \partial A \cap \partial B & \partial A \cap B^{-} \\ A^{-} \cap B^{\circ} & A^{-} \cap \partial B & A^{-} \cap B^{-}\end{array}\right]$, which was proposed by Egenhofer and Franzosa (1991b), Clementini and Di Felice defined a region with a broad boundary, by using two simple regions (Clementini and Di Felice, 1996, 1997). This broad boundary is denoted by $\triangle A$. More precisely, a broad boundary is a simple connected subset of $\mathrm{R}^{2}$ with a hole. Based on empty and non-empty invariance, Clementini and Di Felice's Algebraic model, $R_{9}(A, B)=\left[\begin{array}{ccc}A^{\circ} \cap B^{\circ} & A^{\circ} \cap \Delta B & A^{\circ} \cap B^{-} \\ \Delta A \cap B^{\circ} & \Delta A \cap \Delta B & \Delta A \cap B^{-} \\ A^{-} \cap B^{\circ} & A^{-} \cap \Delta B & A^{-} \cap B^{-}\end{array}\right]$, gave a total of 44 relations between two spatial regions with a broad boundary.

For example, as shown in figure13(a, b), the extended 9-Intersection model proposed by Clementini and Di Felice $(1996,1997)$ yielded the same matrix, $R_{9}(A, B)=\left[\begin{array}{lll}0 & 1 & 1 \\ 1 & 1 & 1 \\ 0 & 1 & 1\end{array}\right]$, that is to say, the topological relations are same, but they are obviously different from each other as shown in figure13(a, b) . Meanwhile, Liu and Shi (2009) proposed a $3 \times 3$ integration model to compute the topological relations between fuzzy line segments, and discovered sixteen topological relations between simple fuzzy region and simple fuzzy line segment. However, the $3 \times 3$ integration model could not get the topological relation as shown in figure 13(c). Then, we will discuss the topological relation as shown in figure 13 of using the new $4 \times 4$ intersection model (equation (1), (6)) in this paper.

We use the new $4 \times 4$ intersection model to obtain the $4 \times 4$ matrix, as follows:

For figure13 (a), the topological relation matrix is: $R(A 1, A 2)=\left[\begin{array}{cccc}1 & 1 & 1 & 1 \\ 1 & 1 & 1 & 1 \\ 1 & 1 & 0 & 0 \\ 0 & 1 & 0 & 0\end{array}\right]$;

For figure13 (b), the topological relation matrix is: $R(A 1, A 2)=\left[\begin{array}{cccc}1 & 1 & 1 & 1 \\ 1 & 1 & 1 & 1 \\ 0 & 1 & 0 & 0 \\ 0 & 1 & 0 & 0\end{array}\right]$;

For figure13 (c), the topological relation matrix is: $R(L 1, A 1)=\left[\begin{array}{llll}0 & 1 & 0 & 0 \\ 0 & 1 & 1 & 1 \\ 1 & 1 & 0 & 0 \\ 1 & 1 & 0 & 0\end{array}\right]$. 


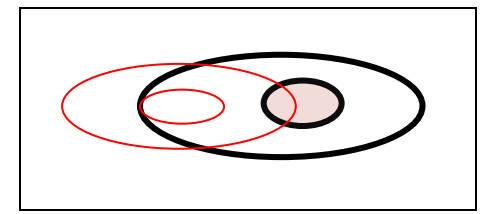

(a)

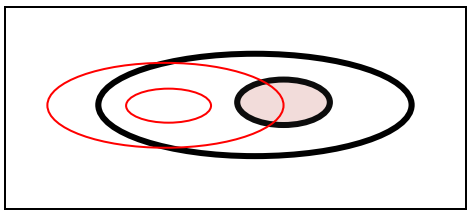

(b)

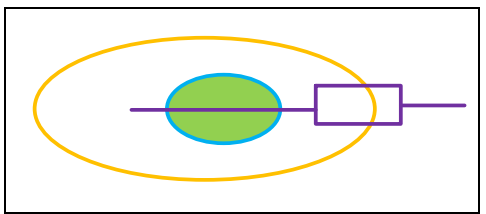

(c)

Figure 13: (a), (b) Two different topological relations between region $A 1$ and $A 2$; (c) Topological relation between a simple fuzzy line segment $L 1$ and a simple fuzzy region $A l$

Through the above comparison analysis, the new proposed model (when taking different values of $\alpha$ and $\beta$ ) not only can compute the topological relations as listed in existing studies (Liu and Shi, 2009; Cohn et al. , 1996, 1997;Clementini and Di Felice,1996,1997), but also the topological relations not currently listed.

\section{Conclusion and Discussion}

Fuzzy topological relations between simple spatial objects can be used for fuzzy spatial queries and spatial analyses. This paper presented a model of fuzzy topological relations for simple spatial objects in GIS. Based on the research of Liu and Shi, we propose a new definition for simple fuzzy line segments and simple fuzzy regions based on the computational fuzzy topology. We also propose a new $4 \times 4$ intersection models to compute the fuzzy topological relations between simple spatial objects, as follows: (1) the topological relations of two simple crisp objects; (2) the topological relations between one simple crisp object and one simple fuzzy object; (3) the topological relations between two simple fuzzy objects. We have discussed some examples to demonstrate the validity of the new model. Through an experiment and comparisons of results, we showed that the proposed method can make finer distinctions, as it is more expressive than the existing fuzzy models.

In this study, fuzzy topology is dependent on the values of $\alpha$ and $\beta$ used in leveling cuts, and different values of $\alpha$ and $\beta$ generate different fuzzy topologies and may have different topological structures. When some applications of fuzzy spatial analyses, an optimal value of $\alpha$ and $\beta$ can be obtained by investigating these fuzzy topologies (Liu and Shi, 2006, Shi and Liu, 2007).

\section{ACKNOWLEDGEMENTS}

The authors would like to thank the Editor and the two anonymous reviewers whose insightful suggestions have significantly improved this letter.

This work was supported by the National Natural Science Foundation of China (No. 41201395, 41161069). Science and Technology Project of Jiangxi Provincial Education Department (No. GJJ14479).

Bol. Ciênc. Geod., sec. Artigos, Curitiba, v. 21, nº 2, p.389-408, abr-jun, 2015. 


\section{REFERENCES}

Chang, C.L. "Fuzzy topological spaces." Journal of Mathematical Analysis and Applications, (24), 182-190, 1968.

Clementini, E., Di Felice, D.P. "An algebraic model for spatial objects with indeterminate boundaries.” In: Burrough, P.A., Frank, A.U. (Eds.), Geographic Objects with Indeterminate Boundaries. Taylor \& Francis, London and Bristol, 155-169, 1996a.

Clementini, E., Sharma, J., Egenhofer, M.J. "Modeling topological spatial relations: Strategies for query processing." Computers and Graphics, 18(6), 815-822, 1994.

Clementini, E., Di Felice, P. "A model for representing topological relationships between complex geometric features in spatial databases." Information Sciences, 90(1), 121-136, 1996b.

Clementini, E., Di Felice, P. “Approximate topological relations." International Journal of Approximate Reasoning, 16(2), 173-204, 1997.

Clementini, E., Di Felice, P., Koperski, K. "Mining multiplelevel spatial association rules for objects with a broad boundary." Data \& Knowledge Engineering, 34(3), 251-270, 2000.

Cohn, A.G., Gotts, N.M. "The 'egg-yolk' representation of regions with indeterminate boundaries." In: Burrough, P.A., Frank, A.U. (Eds.), Geographic Objects with Indeterminate Boundaries, Taylor \& Francis, London and Bristol, 171-187, 1996.

Cohn, A.G., Bennett, B., Gooday, J., Gotts, N.M. "Qualitative spatial representation and reasoning with the region connection calculus." GeoInformatica, 1(1), 1-44, 1997.

Dilo, A., de By, R.A., Stein, A. "A system of types and operators for handling vague spatial objects.” International Journal of Geographical Information Systems, 21(4), 397-426, 2007.

Du, S.H., Qin, Q.M., Wang, Q., Ma, H. "Evaluating structural and topological consistency of complex regions with broad boundaries in multi-resolution spatial databases." Information Sciences, 178(1), 52-68, 2008a.

Du, S.H., Guo,L., Wang, Q., Qin, Q.M. "Efficiently computing and deriving topological relation matrices between complex regions with broad boundaries." ISPRS Journal of Photogrammetry \& Remote Sensing, (63), 593-609, 2008 b.

Du, S.H., Qin, Q.M., Wang, Q. "Fuzzy description of topological relations I: a unified fuzzy 9-intersection model." In: Wang, L., Chen, K., Ong, Y.S. (Eds.), Advances in Natural Computation, Lecture Notes in Computer Science, 3612, 1260-1273, 2005 a.

Du, S.H., Qin, Q.M., Wang, Q. "Fuzzy description of topological relations II: computation methods and examples." In: Wang, L., Chen, K., Ong, Y.S. (Eds.), Advances in Natural Computation, Lecture Notes in Computer Science, 3612, 1274-1279, 2005 b.

Egenhofer, M., Franzosa, R. "Point-set topological spatial relations." International Journal of Geographical Information Systems, 5(2), 161-174, 1991 a.

Egenhofer M, Herring J. "Categorizing Binary Topological Relationships between Regions, Lines, Points in Geographic Databases." Oronoi: Technical Report, Department of Surveying Engineering, University of Maine, Oronoi, ME, $1991 \mathrm{~b}$.

Egenhofer, M., Franzosa, R. "On the equivalence of topological relations." International Journal of Geographical Information Systems, 8(6), 133-152, 1994.

Egenhofer, M., Mark, D. "Modeling Conceptual Neighborhoods of Topological Line-Region Relations." International Journal of Geographical Information Systems, 9(5), 555-565, 1995. 
Egenhofer, M.J. "Query processing in spatial-query-by-sketch.” Journal of Visual Languages and Computing, 8(4), 403-424, 1997.

Li, C.M., Chen, J., Li, Z.L. "Raster-based method or the generation of Voronoi diagrams for spatial entities." International Journal of Geographical Information Systems, 13(3), 209, 1999.

Long Z.G, Li S.Q. "A complete classification of spatial relations using the Voronoi-based nine-intersection model." International Journal of Geographical Information Science, 27(10), 2006-2025, 2013.

Liu, K.F., Shi, W.Z. "Computation of fuzzy topological relations of spatial objects based on induced fuzzy topology." International Journal of Geographical Information Systems, 20(8), 857-883, 2006.

Liu, K.F., Shi, W.Z. "Quantitative fuzzy topological relations of spatial objects by induced fuzzy topology." International Journal of Applied Earth Observation and Geoinformation, (11), 38-45, 2009.

Liu, K.F., Shi, W.Z., Zhang H. "A fuzzy topology-based maximum likelihood classification.” ISPRS Journal of Photogrammetry and Remote Sensing, (66), 103-114, 2011.

Liu, Y.M., Luo, M.K. "Fuzzy Topology.” Singapore: World Scientific, 1997.

Paiva, J.A.C. "Topological equivalence and similarity in multi-representation geographic databases." Ph.D. Dissertation. Department of Surveying Engineering, University of Maine, 1998.

Randell D.A., Cui Z., Cohn A.G. "A spatial logic based on regions and connection." In Proceedings 3rd International Conference on Knowledge Representation and Reasoning, 25-29 October 1992, San Mate, M. Kaufmann, California, 165-176, 1992.

Shi, W.Z., Liu, K.F. "Modelling fuzzy topological relations between uncertain objects in GIS. "Photogrammetric Engineering and Remote Sensing, 70(8), 921-930, 2004.

Shi, W.Z., Liu, K.F. "A fuzzy topology for computing the interior, boundary, and exterior of spatial objects quantitatively in GIS." Computer and Geoscience, 33(7), 898-915, 2007.

Shi, W.Z., Liu, K.F., Huang C.Q. “A Fuzzy-Topology-Based Area Object Extraction Method.” IEEE Transactions on Geoscience and Remote Sensing, 48(1), 147-154, 2010.

Tang, X.M., Kainz, W. "Analysis of topological relations between fuzzy regions in general fuzzy topological space.” In: Proceedings of Spatial Data Handling Conference, Ottawa, Canada, 2002.

Tang, X.M., Kainz, W., Yu F. "Reasoning about changes of land covers with fuzzy settings." International Journal of Remote Sensing, 26(14), 3025-3046, 2005.

Tang, X.M. "Spatial object modeling in fuzzy topological spaces: with applications to land cover change." Ph.D. Dissertation. University of Twente, 2004.

Winter, S. "Uncertain topological relations between imprecise regions." International Journal of Geographical Information Science, 14(5), 411-430, 2000.

Wong, C.K. "Fuzzy points and local properties of fuzzy topology." Journal of Mathematical Analysis and Applications, (46), 316-328, 1974.

Wu, G., Zheng, C. "Fuzzy boundary and characteristic properties of order homomorphisms." Fuzzy Sets and Systems, (39), 329-337, 1991.

Zadeh, L.A. "Fuzzy Sets.” Information and Control, (8), 338-353, 1965.

Recebido em Outubro de 2014. Aceito em Março de 2015.

Bol. Ciênc. Geod., sec. Artigos, Curitiba, v. 21, no 2, p.389-408, abr-jun, 2015. 